\title{
Manejo del aneurisma de la aorta abdominal: Estado actual, evidencias y perspectivas para el desarrollo de un programa nacional
}

\author{
Leopoldo Mariné $M^{1}$, Francisco Valdés $E^{1}$, Renato Mertens $M^{1}$, \\ Albrecht Krämer Sch ${ }^{1}$, Michel Bergoeing $R^{1}$, Dixiana Rivera D ${ }^{2}$, \\ Jeanette Vergara $\mathbf{G}^{\mathrm{a}}$, Claudia Carvajal $\mathrm{N}^{\mathrm{a}}$.
}

O pen and endovascular surgery for the treatment of abdominal aortic aneurism. Review of the available evidence

Open and endovascular surgery are therapeutic alternatives for the treatment of abdominal aortic aneurism. The development of guidelines for its treatment requires a thorough analysis of available evidence to recommend the best treatment for each country's reality. Prospective randomized trials have shown best initial results with endovascular surgery, with higher hospital costs than open surgery. The requirement of anatomical suitability for the placement of endovascular prostheses limits the universal use of endovascular surgery. Moreover, this type of surgery needs a strict imaging and clinical follow up due to the high rates of late complications, which range from $20 \%$ to $40 \%$. Many of these complications require further surgical interventions, elevating costs of treatment. The initial benefit of endovascular surgery is lost during long follow up as survival curves become similar to those of open surgery. Even for patients with a high surgical risk, the benefits of endovascular surgery are doubtful (Rev Méd Chile 2009; 137: 1081-8).

(Key words: Aortic aneurysm, abdominal; Blood vessel prothesis; Surgical procedures, operative)

Recibido el 7 de agosto, 2008. Aceptado el 20 de octubre, 2008.

${ }^{1}$ Departamento de Cirugía Vascular y Endovascular, División de Cirugía, Pontificia Universidad Católica de Chile, Santiago de Chile. ${ }^{2}$ Instituto Vascular de Santiago, Santiago de Chile

${ }^{a}$ Enfermera Universitaria

$\mathrm{E}$ 1 desarrollo de técnicas endovasculares en el manejo de la patología arterial ha permitido su aplicación en distintos territorios incluyendo a la

Correspondencia a: Dr Leopoldo Mariné M. Apoquindo 3990, Of. 601, Santiago. Fax (562) 6326812.

E mail: marinepolo@yahoo.com aorta. El tratamiento endovascular de la aorta, luego de un explosivo desarrollo inicial, en los últimos años ha fallado en reemplazar a la cirugía tradicional debido a su alto costo y a la frecuente necesidad de reintervenciones posteriores. Nuestra experiencia ha sido concordante con la de grandes centros internacionales, compartiendo las 
mismas inquietudes respecto al espacio que esta técnica debe ocupar en el arsenal terapéutico. Al momento de establecer cuáles deberían ser las normas nacionales de tratamiento de los aneurismas de la aorta abdominal, se deben tomar en cuenta todas las variables en juego. A continuación expondremos algunas características básicas de esta patología, el valor del tamizaje, las distintas perspectivas de análisis de tratamiento tradicional y endovascular, los grandes estudios prospectivos y randomizados, los resultados iniciales y alejados y los análisis de costos que, en conjunto, fundamentan el planteamiento terapéutico que han seguido numerosos países, que también hemos adoptado en nuestro centro y que expondremos en el final de esta revisión.

El aneurisma de la aorta abdominal (AAA) se caracteriza por alteraciones estructurales de la pared arterial que determinan su debilitamiento, facilitando la dilatación progresiva, la que puede provocar muerte por ruptura. Esta ominosa historia natural es modificable.

El enfrentamiento terapéutico debe iniciarse con la pesquisa en pacientes asintomáticos por tamizaje de la población en riesgo, en especial hombres mayores de 65 años en quienes se ha demostrado menor mortalidad con el diagnóstico precoz $^{1,2}$. En AAA pequeños el manejo consiste en el control de factores de riesgo y seguimiento ecográfico del diámetro aórtico.

La reparación está indicada si el diámetro del AAA es mayor o igual a $5 \mathrm{~cm}$, en AAA de crecimiento rápido $(>0,5 \mathrm{~cm}$ al año) o por aparición de síntomas. La cirugía electiva tiene mortalidad dramáticamente inferior a la operación en condiciones de emergencia. La mortalidad operatoria de la cirugía electiva del AAA es 0,73 a 5\%3,4, comparada con la mortalidad prehospitalaria del AAA roto de $50 \%$ a $60 \%$ y mortalidad operatoria de 25\%-60\% en los pacientes que llegan a ser tratados, alcanzando a 100\% sin intervención.

En Chile desconocemos la tasa de muerte por AAA roto o el número de AAA operados ya sea electivamente o por ruptura. Un estudio en nuestra población 5 muestra una frecuencia de 5,9\% (7,6\% en hombres y $1,1 \%$ en mujeres) en personas mayores de 60 años con al menos un factor de riesgo cardiovascular.

Debemos establecer una estrategia de manejo del AAA adecuada, la que debe incluir: diagnóstico precoz y tratamiento electivo apropiado, efec- tuado en condiciones de la menor morbimortalidad posible y de forma costo-efectiva.

Desde los años 1990-99 además de la cirugía abierta (CA), que elimina el AAA a través de una laparotomía, disponemos de una nueva modalidad terapéutica: la cirugía endovascular (CE) que consiste en la exclusión in toto del AAA mediante una endoprótesis introducida y desplegada por vía transfemoral. En comparación con la CA, la CE tiene la ventaja de ser mínimamente invasiva, lo que se traduce en menor morbimortalidad perioperatoria y una estadía hospitalaria más breve. Sin embargo, conlleva un mayor costo de realización, además de la necesidad de seguimiento de por vida con imágenes (tomografía axial multicorte en fase arterial), con la probabilidad de fallas tardías del dispositivo que requieren de reintervención con costos adicionales en $20 \%$ a $40 \%$ de los pacientes ${ }^{4,6}$.

Al momento de definir una guía para el manejo terapéutico del AAA, es imperativo un análisis racional de la evidencia disponible para seleccionar la mejor forma de tratamiento desde la perspectiva de las condiciones socioeconómicas y culturales de nuestro país.

El análisis de este tema varía según la perspectiva de los distintos actores involucrados.

Perspectiva del paciente. Sus motivaciones son: el riesgo quirúrgico, posibles complicaciones, magnitud del dolor, retorno rápido a su hogar o al trabajo y eventuales costos. En el enfrentamiento inicial, el seguimiento postoperatorio y las reintervenciones tienen una prioridad secundaria respecto de las anteriores.

Perspectiva del cirujano. La CE es una alternativa limitada ya que la formación y las habilidades requeridas son distintas a las de la $\mathrm{CA}$. El cirujano debe demostrar un entrenamiento acreditado, conociendo los distintos dispositivos disponibles y sus limitaciones, de manera de ofrecer al paciente similar seguridad con CE y con CA, es decir, con una morbimortalidad dentro de los estándares internacionales. A su vez el equipo tratante debe mantener un volumen de intervenciones suficiente considerando que los mejores resultados son función del mayor número de procedimientos realizados ${ }^{7-11}$.

Perspectivas de la industria. Los fabricantes de endoprótesis financian la investigación para dise- 
ñar nuevos y mejores dispositivos, realizando frecuentes modificaciones en busca de disminuir sus potenciales fallas y así prolongar su durabilidad. Además financian estudios clínicos para demostrar la aplicabilidad y lograr su aprobación para el uso en humanos. Desde el punto de vista comercial, realizan publicidad y mercadeo promoviendo su producto. Esta "inversión" es traspasada al paciente elevando el costo del dispositivo, siendo necesario un volumen de ventas que permita la sustentabilidad de la empresa. Por otro lado, algunos incluyen en el precio del dispositivo, el "entrenamiento" provisto por ellos para los operadores ocasionales, generando un sesgo importante al momento de seleccionar la endoprótesis, pudiendo primar compromisos comerciales sobre consideraciones técnicas.

Perspectiva del hospital. Salvo que el costo de la endoprótesis y del procedimiento sea por cuenta del hospital, la CE es mejor evaluada por cualquier administración hospitalaria respecto de la $\mathrm{CA}$, al requerir menos días de hospitalización. Sin embargo, debe considerarse que para que sea posible realizar CE con estándares apropiados de seguridad, el hospital debe cumplir con estrictos requerimientos de planta física y recursos humanos, entre otros: tomografía axial multicorte y radiólogos preparados accesibles permanentemente, angiógrafo adecuado en ambiente de esterilidad quirúrgica, personal de apoyo de radiología entrenado, unidad de tratamiento intensivo y otros. Hoy por hoy, en nuestro país, lo anterior implica necesariamente la concentración de estos casos en un número restringido de centros públicos y privados.

Perspectiva de las Sociedades Científicas. En su rol de divulgar los conocimientos y la tecnología aplicada, las Sociedades deben sugerir estándares y aportar nuevas evidencias, permaneciendo en un punto neutro y crítico. La visión respecto a la CE y la CA debe ser independiente de los intereses individuales del paciente, del cirujano, del hospital o de la industria.

Perspectiva del país. Esta debe seguir una sola lógica: el mejor tratamiento disponible, que logre la meta de disminuir morbimortalidad de la patología, con una relación costo-beneficio favo- rable, en este caso aplicable a un segmento pasivo de la población donde la rentabilidad social de lo invertido está limitada por la menor esperanza de vida de los pacientes a tratar.

La evidencia más relevante proviene de los estudios multicéntricos, prospectivos y randomizados que comparan CA con CE. Existen dos trabajos efectuados en pacientes con riesgo quirúrgico habitual o estándar: el EVAR-1 (Reino Unido) $^{12}$ y el DREAM (Holanda) ${ }^{13}$, que publican sus resultados a $4^{14}$ y $2^{15}$ años. En pacientes de alto riesgo quirúrgico existe un solo estudio prospectivo que en forma aleatoria compara tratamiento con CE vs observación (EVAR-2) ${ }^{16}$.

Hay dos publicaciones del sistema de veteranos en Estados Unidos de Norteamérica (EE.UU.) (VA) ${ }^{17,18}$ que comparan ambos tratamientos en forma retrospectiva los que, junto a series clínicas provenientes de instituciones individuales, deben ser interpretados en forma cuidadosa, con un nivel de evidencia menor.

\section{Resumen de la evidencia}

1. Los resultados clínicos iniciales de CE son mejores. La mayoría de los estudios confirman menor morbimortalidad, permanencia en unidad de tratamiento intensivo, necesidad de transfusiones y estada hospitalaria global para $\mathrm{CE}^{12,13,17}$.

2. El beneficio de menor morbimortalidad de la CE respecto a CA es sólo inicial, ya que en el mediano plazo las curvas de sobrevida se equiparan. En el estudio EVAR-1 la mejor sobrevida inicial a 30 días en pacientes tratados con CE no se traduce en una mayor sobrevida posterior (4 años). Igualmente, la mejoría inicial de la calidad de vida se pierde antes del primer año de seguimiento ${ }^{14}$. El estudio DREAM mostró similares resultados: a dos años la tasa de sobrevida fue de $89,7 \%$ para CE y de $89,6 \%$ para CA $(P=0,86)$. Las muertes atribuibles al AAA fueron de 5,7\% para CA y de $2,1 \%$ para CE $(P=0,05)$, la diferencia de $3,7 \%$ fue explicada de igual manera al atribuirse solamente a la ventaja de la mortalidad perioperatoria inicial y no a un posterior beneficio del $\mathrm{CE}^{15}$.

3. La CE no es universal. La aorta, arterias ilíacas y femorales deben permitir el avance del dispositivo dentro de ellas y presentar sectores de arteria sana adyacentes al AAA apropiadas 
para el anclaje de la endoprótesis, asegurando el total sellamiento y exclusión del AAA. En la Tabla 1 detallamos las situaciones anatómicas que limitan la realización de la CE. Entre 34\% y $46 \%$ de los pacientes no presentan los criterios anatómicos mínimos para $\mathrm{CE}^{6,19,20}$. Los pacientes de alto riesgo quirúrgico, teóricamente los que se podrían beneficiar con $\mathrm{CE}$, suelen presentar mayor limitación anatómica y sólo $49 \%$ cumple criterios anatómicos ${ }^{19}$. Si se evalúa por género, $70 \%$ de los hombres y sólo $40 \%$ de las mujeres cumplen dichos criterios $^{19}$.

4. En pacientes de alto riesgo, el beneficio de la CE respecto a la sola observación es controvertido. El EVAR-2 randomizó pacientes calificados de "alto riesgo" para CA, entre CE y no tratamiento quirúrgico alguno, concluyendo que CE no es mejor que la sola observación. Si bien se ha criticado la validez de este protocolo, al momento es el único estudio prospectivo y randomizado al respecto. Este trabajo no favorece la CE al fallar en demostrar mejor sobrevida global o libre de ruptura del AAA incurriendo en un costo muy superior ${ }^{16}$. Según los criterios definidos en la Tabla 2, alrededor de $22 \%$ y $24,5 \%$ de los pacientes con AAA serían de alto riesgo para $\mathrm{CA}^{12,21}$, siendo el principal riesgo de naturaleza cardiaca.

Recientemente, un estudio retrospectivo norteamericano $^{21}$ proveniente de 5 centros, compara la $\mathrm{CE}$ con la $\mathrm{CA}$ en pacientes de alto riesgo,

\section{Tabla 1. C riterios anatómicos que contradicen el uso de tratamiento endovascular de aneurismas de la aorta abdominal ${ }^{19}$}

- Cuello aórtico de diámetro >28 mm

- Cuello aórtico de largo $<15 \mathrm{~mm}$

- Cuello aórtico de angulación >60응

- Tortuosidad ilíaca severa

- Trombo mural extenso en cuello aórtico

- Diámetro de arteria de acceso (ilíaca) $<7 \mathrm{~mm}$

- Diámetro de bifurcación aórtica <18 mm

- Aneurismas ilíacos comunes bilaterales que requieren de cobertura de ambas arterias hipogástricas

- Arteria renal accesoria esencial con seguimiento promedio de 2,5 años. En este estudio los beneficios de una menor mortalidad inicial de CE $(2,9 \%$ vs $5,1 \%)$ se pierden más allá de 30 días, siendo la sobrevida a 4 años de 56\% para CE y de 66\% para CA ( $\mathrm{p}=0,23)$. La mortalidad a 30 días en pacientes de alto riesgo con CE fue de 2,9\% comparado con $9 \%$ reportado en EVAR-2. Por otro lado, la mortalidad en CA $(5,1 \%)$ es baja considerando el riesgo de la población en estudio. Sin embargo, hay múltiples publicaciones de pacientes catalogados dentro de este grupo de riesgo que reportan muy buenos resultados con $\mathrm{CA}^{22,23}$.

El autor principal de los estudios EVAR sugiere que la CE puede beneficiar a los pacientes de alto riesgo quirúrgico en los cuales sus comorbilidades puedan ser mejoradas, con mayor expectativa de vida ${ }^{24}$. Sin embargo, según otros autores, la baja sobrevida de algunos pacientes de alto riesgo quirúrgico hace al menos discutible su tratamiento con CE y la simple observación aparece como una buena alternativa $^{23}$.

\section{Tabla 2. Criterios de alto riesgo quirúrgico para cirugía abierta tradicional ${ }^{23}$}

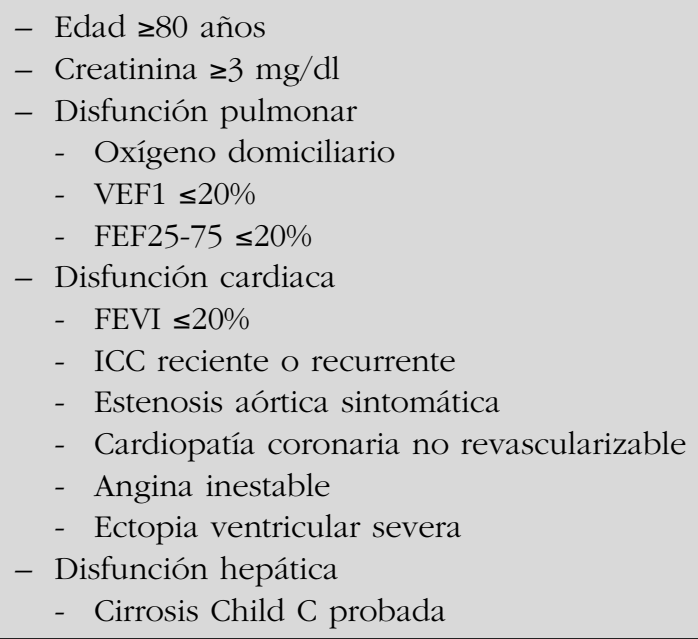

VEF1: Volumen espiratorio forzado del $1^{\mathrm{er}}$ segundo; FEF25-75: Flujo espiratorio forzado entre 25\% y $75 \%$ de la capacidad vital; FEVI: Fracción de eyección del ventrículo izquierdo; ICC: Insuficiencia cardiaca congestiva. 
5. El costo hospitalario de CE es mayor que CA. El costo de la CE es significativamente mayor como lo muestra el estudio EVAR-1 ${ }^{14}$, estudios multicéntricos ${ }^{25}$ y estudios en grandes centros médicos ${ }^{26-28}$, fluctuando entre $12 \%$ y $72 \%$ más caro que $\mathrm{CA}^{6}$. Este mayor costo total supera los beneficios económicos de menores estadías en intensivo o días de hospitalización ${ }^{25-28}$. La Cleveland Clinic estimó el mayor costo hospitalario de CE en US\$ 7.205 por paciente ${ }^{26}$. El alto costo de la endoprótesis es el responsable de más de la mitad del costo del $\mathrm{CE}^{25}$. Comparada con la prótesis tradicional utilizada en CA, la endoprótesis para el CE es 22 veces más cara ${ }^{28}$. A nivel nacional esta diferencia es aún mayor.

6. La CE demanda frecuentes reintervenciones durante el seguimiento. En EVAR-1 la CE presenta 5 veces más complicaciones que la CA a 4 años (17,6 vs 3,3 por 100 personas año, p $<0,0001$ ), con al menos una reintervención en $20 \%$ de los CE vs $6 \%$ en los $\mathrm{CA}^{14}$. En el estudio DREAM la tasa de reintervención en CE fue casi 3 veces más que CA en los primeros 9 meses ( $p$ $<0,03)^{15}$. EUROSTAR, registro europeo de CE en AAA, comunicó tasas de reintervención de $10,3 \%, 33 \%$ y $38 \%$ a 1,3 y 4 años de seguimiento. Las fallas fueron resueltas en $76 \%$ con CE, $12 \%$ con CA sobre la aorta y $11 \%$ con puentes extraanatómicos ${ }^{29}$. La Clínica Mayo reportó evolución libre de reintervención en el primer año post-CE de $65 \%$ y de $93 \%$ para CA, afirmando que esta información debe considerarse al momento de ofrecerle $\mathrm{CE}^{30}$. En una publicación del EUROSTAR se comunica que los AAA con diámetro $\geq 5,5 \mathrm{~cm}$ tratados con CE presentaron una tasa anual de muerte relacionada al aneurisma entre $0,3 \%$ y $1 \%$ los primeros tres años de seguimiento, la que aumentó entre $2,1 \%$ y $8 \%$ al cuarto año, por lo que sugieren incrementar y no disminuir la frecuencia de controles, por lo que en la práctica, el paciente nunca se puede considerar "curado" de su enfermedad ${ }^{31}$. Según EUROSTAR, el riesgo acumulado de ruptura del AAA post-CE es de 1\% anual. El 86\% de los AAA tratados con CE complicados de ruptura tardía pudo ser operado, con $41,6 \%$ de mortalidad, y una mortalidad final de todos los pacientes complicados de ruptura tardía de $64,5 \%$. Otra variable es la tasa de reintervenciones por falla alejada de la endoprótesis, situación grave. En manos expertas la tasa de reintervención es de $15 \%$, con una mortalidad de $8 \%$, aumentando ésta a $18 \%$ si la reintervención requiere de $\mathrm{CA}$. Al analizar los factores predictores de reintervención, los autores reconocen la inclusión de pacientes en el límite de la indicación para CE, por anatomía del AAA desfavorable para la técnica ${ }^{32}$. La única experiencia nacional reporta un éxito técnico de 98,8\%, mortalidad a 30 días de 1,3\%, sobrevida a 5 años libre de ruptura de 100\%, sobrevida a 4 años libre de complicaciones de $82,7 \%$, resultados comparables con series extranjeras, producto de la rigurosidad en la selección de los casos, en la técnica prolija empleada y en el seguimiento estrecho de los pacientes $^{33}$.

7. El costo de CE aumenta por los costos aditivos del seguimiento y reintervenciones. EVAR-1, en su seguimiento a 4 años ${ }^{14}$, reportó un costo global acumulado de CE: UK£13.257 vs £9.946 de CA (diferencia de $\{3.311$ ). Otras publicaciones afirman que el costo del seguimiento debería ser agregado en el análisis de comparación entre estas dos modalidades terapéuti$\operatorname{cas}^{34,35}$. En un análisis similar sobre costos en CE, Noll determinó que el monto acumulado sólo por el seguimiento a 5 años alcanza US\$ 11.351 , esto es $44 \%$ de aumento del costo global. Más aún, el costo de seguimiento con reintervención (US\$ 31.696) es 8,6 veces mayor que cuando no se requiere reintervención (US\$ 3.668, p <0,05) 36 .

8. Recomendaciones de Agencias Sanitarias y Sociedades Científicas. En una extensa revisión, cuyo objetivo fue entregar información para ayudar a médicos y empleados encargados de políticas en la toma de decisiones acerca de la provisión de los sistemas de salud en EE.UU., la Agency for Healthcare Research and Quality del U.S. Department of Health and Human Services ${ }^{6}$ concluye que: 1) a pesar de resultados iniciales favorables, la CE no ha demostrado mejor sobrevida alejada que la CA, 2) tampoco ha demostrado mejor sobrevida en pacientes de alto riesgo no aptos para CA y 3) se asocia a más complicaciones, necesidad de reintervenciones, monitorización y costos, comparados con la CA o la no intervención. 
En una segunda revisión proveniente del American College of Cardiology, la American Heart Association y la Society for Vascular Surgery, entre otros ${ }^{4}$, se establecen las siguientes recomendaciones:

Clase I: en pacientes con riesgo quirúrgico bajo está indicada la CA.

Clase IIb: en pacientes con un riesgo quirúrgico alto la CE es una opción razonable.

Finalmente, la Sociedad Española de Angiología y Cirugía Vascular comparte las recomendaciones anteriores ${ }^{37}$

9. Futuro. El rol de la $\mathrm{CE}$ en el tratamiento de los AAA debe ser reevaluado a la luz de los estudios prospectivos y randomizados en curso (estudio ACE en Francia o el estudio OVER en EE.UU.). Mientras tanto, la evidencia aquí analizada ha sido considerada en el diseño de políticas públicas en países como Bélgica, donde el Ministerio de Salud limitó el financiamiento de la CE a circunstancias de excepción ${ }^{38}$.

A nuestro juicio, tres condicionantes son fundamentales para realizar CE de la aorta y constituyen, a su vez, limitantes desde la perspectiva del país:

1. Resultados. La morbimortalidad a 30 días con CE debe ser menor que con CA. La obtención de buenos resultados en CE es multifactorial, siendo cada eslabón tan importante como el adyacente: entrenamiento del cirujano, volumen operatorio y complejidad técnica, buenos estudios radiológicos pre y post intervención, selección de endoprótesis, infraestructura adecuada y habilitada permanentemente, entre otros. En países desarrollados esto sólo se logró después de 10 años de establecida la técnica, considerando que tienen posibilidades de pagar altos costos de investigación, desa-

\section{REFERENCIAS}

1. Cosford PA, Leng GC. Screening for abdominal aortic aneurysm. Cochrane Database Syst Rev 2007; 2: $1-17$.

2. Fleming C, Whitlock EP, Beil Tl, Lederle FA. Screening for abdominal aortic aneurysm: a best-evidence systematic review for the U.S. Preventive Services Task Force. Ann Intern Med 2005; 142: 203-11. rrollo tecnológico y con gastos de salud hasta $16 \%$ del PIB.

2. Resolución. En los AAA de más de $5 \mathrm{~cm}$ el tratamiento no debe tener retraso. Es imperativa la disponibilidad y accesibilidad hospitalaria, con recursos humanos y físicos permanentes, para evitar el riesgo de ruptura durante el periodo en "lista de espera", fenómeno lamentable, observado en estudios británicos.

3. Compromiso. Mantener un seguimiento de los pacientes estrecho y de por vida, con financiamiento de todas las reintervenciones necesarias, independientemente de su costo. En el caso de complicaciones tardías, eventuales limitaciones de cobertura económica para reintervenciones dejarían al paciente CE expuesto a muerte por ruptura del AAA.

En conclusión, de acuerdo con la información expuesta, el beneficio de CE implica un incremento de los costos hospitalarios de al menos 33\% sin un beneficio mantenido de la calidad de vida ni de sobrevida global a 4 años ${ }^{20}$. Por costo y ausencia de diferencia en los resultados alejados, la CA continúa siendo la primera alternativa para pacientes portadores de AAA con indicación de tratamiento electivo.

La CE tiene indicación en pacientes con anatomía del AAA favorable, asintomáticos, con mayor riesgo quirúrgico objetivo, portadores de patologías asociadas susceptibles de mejoría con tratamiento médico, con expectativa de vida de al menos 5 años y capaces de iniciar un seguimiento estricto.

La generación de registros y el análisis de los resultados obtenidos es imprescindible a la hora de reevaluar los resultados de las diferentes formas de tratamiento en nuestro ambiente nacional.

3. Valdés F, Krämer A, Mertens R, Santini A, Canessa R, LEMA G ET AL. Aneurisma aórtico abdominal: evolución de la morbimortalidad de la cirugía electiva durante 20 años. Rev Méd Chile 1997; 125: 425-32.

4. Hirsch at, Haskal ZJ, Hertzer NR, Bakal CW, Creager MA, Halperin JL et al. ACC/AHA 2005 Practice Guidelines for the management of patients with peripheral arterial disease (lower extremity, renal, mesenteric and abdominal aortic): a collaborative 
report from the American Association for Vascular Surgery/Society for Vascular Surgery, Society for Cardiovascular Angiography and Interventions, Society for Vascular Medicine and Biology, Society of Interventional Radiology, and the ACC/AHA Task Force on Practice Guidelines (Writing Committee to Develop Guidelines for the Management of Patients With Peripheral Arterial Disease): endorsed by the American Association of Cardiovascular and Pulmonary Rehabilitation; National Heart, Lung, and Blood Institute; Society for Vascular Nursing; TransAtlantic Inter-Society Consensus; and Vascular Disease Foundation. Circulation 2006; 113: e463-654.

5. Valdés F, Sepúlveda N, Krämer A, Mertens R, Bergoeing M, Mariné L et al. Frecuencia de aneurisma aórtico abdominal en población adulta con factores de riesgo conocidos. Rev Méd Chile 2003; 131: 741-7.

6. Wilt TJ, Lederle FA, MacDonald R, Jonk YC, Rector TS, KANE RL. Comparison of endovascular and open surgical repairs for abdominal aortic aneurysm. Evid Rep Technol Assess 2006; 144: 1-113.

7. Hannan El, Kilburn H JR, O'Donnell JF, Bernard HR, SHIELDS EP, LINDSEY ML ET AL. A longitudinal analysis of the relationship between in-hospital mortality in New York State and the volume of abdominal aortic aneurysm surgeries performed. Health Serv Res 1992; 27: 517-42.

8. Kazmers A, Jacobs L, Perkins A, Lindenauer SM, Bates E. Abdominal aortic aneurysm repair in Veterans Affairs medical centers. J Vasc Surg 1996; 23: 191-200.

9. Birkmeyer JD, Dimick JB, Staiger DO. Operative mortality and procedure volume as predictors of subsequent hospital performance. Ann Surg 2006; 243: 411-7.

10. Lobato AC, Rodríguez-López J, Diethrich EB. Learning curve for endovascular abdominal aortic aneurysm repair: evaluation of a 277-patient single-center experience. J Endovasc Ther 2002; 9: 262-8.

11. Dimick JB, Upchurch GR Jr. Endovascular technology, hospital volume, and mortality with abdominal aortic aneurysm surgery. J Vasc Surg 2008; 47: 1150-4.

12. Greenhalgh RM, Brown LC, Kwong GP, Powell JT, Thompson SG, Evar Trial Participants. Comparison of endovascular aneurysm repair with open repair in patients with abdominal aortic aneurysm (EVAR trial 1), 30-day operative mortality results: randomized controlled trial. Lancet 2004; 364: 843-8.

13. Prinssen M, Verhoeven El, Buth J, Cuypers PW, Van Sambeek MR, Balm R et al, Dutch Randomized Endovascular Aneurysm Management (Dream) Trial Group. A randomized trial comparing conventional and endovascular repair of abdominal aortic aneurysms. N Engl J Med 2004; 351: 1607-18.

14. EVAR 1 Trial Participants. Endovascular aneurysm repair vs open repair in patients with abdominal aortic aneurysm (EVAR trial 1): randomized controlled trial. Lancet 2005; 365: 2179-86.

15. Blankensteijn JD, De Jong Se, Prinssen M, Van Der Ham aC, Buth J, Van Sterkenburg SM et al, Dutch Randomized Endovascular Aneurysm Management (Dream) Trial Group. Two-year outcomes after conventional or endovascular repair of abdominal aortic aneurysms. N Engl J Med 2005; 352: 2398-405.

16. EVAR 2 Trial Participants. Endovascular aneurysm repair and outcome in patients unfit for open repair of abdominal aortic aneurysm (EVAR trial 2): randomized controlled trial. Lancet 2005; 365: 2187-92.

17. Bush RL, Johnson ML, Collins TC, Henderson WG, KHuRI SF, Yu HJ ET AL. Open versus endovascular abdominal aortic aneurysm repair in VA hospitals. J Am Coll Surg 2006; 202: 577-87.

18. Bush RL, Johnson ML, Hedayati N, Henderson WG, Lin $\mathrm{PH}$, Lumsden AB. Performance of endovascular aortic aneurysm repair in high-risk patients: results from the Veterans Affairs National Surgical Quality Improvement Program. J Vasc Surg 2007; 45: 227-233.

19. Carpenter JP, Baum Ra, Barker CF, Golden MA, Mitchell ME, Velázquez OC ET AL. Impact of exclusion criteria on patient selection for endovascular abdominal aortic aneurysm repair. J Vasc Surg 2001; 34: 1050-4.

20. Cronenwett JL. Endovascular aneurysm repair: important mid-term results. Lancet 2005; 365: 2156-8.

21. Sicard GA, Zwolak RM, Sidawy AN, White RA, Siami FS; Society for Vascular Surgery Outcomes CommitTEE. Endovascular abdominal aortic aneurysm repair: long-term outcome measures in patients at high-risk for open surgery. J Vasc Surg 2006; 44: 229-36.

22. Valdés F, Bergoeing M, Krämer A, Mertens R, Canessa R, Lema G et al. Aneurisma aórtico abdominal en pacientes mayores de 80 años: tratamiento quirúrgico convencional en 80 casos consecutivos. Rev Méd Chile 2003; 131: 981-6.

23. Menard MT, Chew DK, Chan RK, Conte MS, Donaldson MC, Mannick JA ET aL. Outcome in patients at high risk after open surgical repair of abdominal aortic aneurysm. J Vasc Surg 2003; 37: 285-92.

24. GreEnhalgh RM. Author reply. Lancet 2005; 366: 891.

25. Sternbergh WC $3^{\text {rd }}$, Money SR. Hospital cost of endovascular versus open repair of abdominal aortic aneurysms: a multicenter study. J Vasc Surg 2000; 31: 237-44.

26. Clair DG, Gray B, O'Hara PJ, Ouriel K. An evaluation of the costs to health care institutions of endovascular aortic aneurysm repair. J Vasc Surg 2000; 32: 148-52.

27. Dryjski M, O’Brien-Irr MS, Hassett J. Hospital costs for endovascular and open repair of abdominal aortic aneurysm. J Am Coll Surg 2003; 197: 64-70.

28. Angle N, Dorafshar AH, Moore WS, Quiñones-BaldriCh WJ, Gelabert HA, Ahn SS et al. Open versus 
endovascular repair of abdominal aortic aneurysms: what does each really cost? Ann Vasc Surg 2004; 18 : 612-8.

29. Laheij RJ, Buth J, Harris Pl, Moll FL, Stelter WJ, VerhoEven EL. Need for secondary interventions after endovascular repair of abdominal aortic aneurysms. Intermediate-term follow-up results of a European collaborative registry (EUROSTAR). Br J Surg 2000; 87: 1666-73.

30. Elkouri S, Gloviczki P, McKusick Ma, Panneton JM, Andrews J, Bower TC et al. Perioperative complications and early outcome after endovascular and open surgical repair of abdominal aortic aneurysms. J Vasc Surg 2004; 39: 497-505.

31. Harris PL, Buth J. An update on the important findings from the EUROSTAR EVAR registry. Vascular 2004; 12: 33-8.

32. Sampram ES, Karafa MT, Mascha EJ, Clair DG, GreenBERG RK, Lyden SP ET AL. Nature, frequency, and predictors of secondary procedures after endovascular repair of abdominal aortic aneurysm. J Vasc Surg 2003; 37: 930-7.

33. Valdés F, Mertens R, Krämer A, Bergoeing M, Mariné L, Canessa R et al. Tratamiento endovascular de aneu- risma aórtico abdominal: resultados en 80 pacientes consecutivos. Rev Méd Chile 2006; 134: 1265-74.

34. Epstein DM, Sculpher MJ, Manca A, Michaels J, Thompson SG, Brown LC ET al. Modelling the longterm cost-effectiveness of endovascular or open repair for abdominal aortic aneurysm. Br J Surg 2008; 95: 183-90.

35. Hayter Cl, Bradshaw SR, Allen RJ, Guduguntla M, Hardman DT. Follow-up costs increase the cost disparity between endovascular and open abdominal aortic aneurysm repair. J Vasc Surg 2005; 42: 912-8.

36. Noll RE Jr, Tonnessen BH, Mannava K, Money SR, STERNBERGH WC $3^{\text {RD }}$. Long-term postplacement cost after endovascular aneurysm repair. J Vasc Surg 2007; 46: 9-15.

37. Maeso-Lebrun J, Clará A, Escudero-Rodríguez JR, Gesto-Castromil R, Gómez-Palonés FJ, Riambau-Alonso V ET AL. Tratamiento endovascular de la patología aneurismática de la aorta abdominal. Angiología 2007; 59: S3-S28.

38. García Martín I. Situación actual de la cirugía endovascular de los aneurismas de la aorta abdominal. Cir Cardiov 2007; 14: 397-400. 